\title{
Relationship between transforming growth factor- $\beta 1$ and type 2 diabetic nephropathy risk in Chinese population
}

\author{
Tianbiao Zhou ${ }^{1 * \dagger}$, Hong-Yan $\mathrm{Li}^{2+}$, Hongzhen Zhong ${ }^{1 \dagger}$ (ID and Zhiqing Zhong ${ }^{1}$
}

\begin{abstract}
Background: Diabetes mellitus (DM) is divided into four different etiological categories: type 1 DM (T1DM), type 2 DM (T2DM), other specific types, and gestational DM. One severe complication of T2DM is type 2 diabetic nephropathy (T2DN). The possible association of serum transforming growth factor- $\beta 1$ (TGF- $\beta 1$ ) levels and the TGF$\beta 1$ T869C gene polymorphism with patient susceptibility to T2DN in Chinese population is unclear at present. This study was conducted to assess these relationships in Chinese population by a meta-analysis.

Methods: Association reports were searched and pulled from the Cochrane Library, the China Biological Medicine Database (CBM), and PubMed on March 1, 2018, and eligible studies were selected and used for calculations. The results were expressed as weighted mean differences (MD) for continuous data. Odds ratios (OR) were used to express the results for dichotomous data. Additionally, 95\% confidence intervals (Cl) were calculated.
\end{abstract}

Results: Forty-eight reports for the relationship between serum TGF- $\beta 1$ levels and the risk of T2DN and 13 studies on the association of the TGF- $\beta 1$ T869C gene polymorphism with susceptibility to T2DN in Chinese population were retrieved from this study. Serum TGF- $\beta 1$ levels in the T2DM group were higher than those in the normal control group ( $M D=17.30,95 \% \mathrm{Cl}: 12.69-21.92, P<0.00001)$. The serum TGF- $\beta 1$ level in the T2DN group was significantly higher than that in the normal control group ( $\mathrm{MD}=70.03,95 \% \mathrm{Cl}: 60.81-79.26, P<0.00001 ;)$. The serum TGF- $\beta 1$ level in the T2DN group was significantly higher than that in the T2DM group (MD $=56.18$, 95\% Cl: 46.9665.39, $P<0.00001$ ). Serum TGF- $\beta 1$ levels in T2DM patients with microalbuminuria were increased when compared with those in T2DM patients with normoalbuminuria. Furthermore, serum TGF- $\beta 1$ levels in T2DM patients with macroalbuminuria were increased when compared with those in T2DM patients with microalbuminuria. The TGF- $\beta 1$ T allele, $T$ allele and CC genotype were associated with T2DN susceptibility in Chinese population ( $T$ : $O R=0.74$, 95\% Cl: 0.59-0.92, $P=0.007 ; T \mathrm{~T}: \mathrm{OR}=0.55,95 \% \mathrm{Cl}: 0.31-0.96, P=0.04 ; C C: \mathrm{OR}=1.38,95 \% \mathrm{Cl}: 1.14-1.67, P=0.001)$.

Conclusions: High levels of TGF- $\beta 1$ are associated with susceptibility to T2DM, T2DN and the progression of proteinuria in T2DN patients in Chinese population. Further, the TGF- $\beta 1 \mathrm{~T}$ allele, and $T$ genotype were protective factors against the onset of T2DN and CC genotype was a risk factor for the susceptibility of T2DN in Chinese populations.

Keywords: Type 2 diabetic nephropathy (T2DN), Diabetes mellitus (DM), Transforming growth factor- $\beta 1$, T869C, Gene polymorphism, Meta-analysis

\footnotetext{
* Correspondence: zhoutb@aliyun.com

${ }^{\dagger}$ Tianbiao Zhou, Hongyan Li and Hongzhen Zhong contributed equally to this work.

${ }^{1}$ Department of Nephrology, the Second Affiliated Hospital of Shantou University Medical College, No 69 Dongsha Road, Shantou 515041, China Full list of author information is available at the end of the article
}

(c) The Author(s). 2018 Open Access This article is distributed under the terms of the Creative Commons Attribution 4.0 International License (http://creativecommons.org/licenses/by/4.0/), which permits unrestricted use, distribution, and reproduction in any medium, provided you give appropriate credit to the original author(s) and the source, provide a link to the Creative Commons license, and indicate if changes were made. The Creative Commons Public Domain Dedication waiver (http://creativecommons.org/publicdomain/zero/1.0/) applies to the data made available in this article, unless otherwise stated. 


\section{Background}

Transforming growth factor beta1 (TGF- 31 ) is one of the pro-fibrotic cytokines and is thought to be the primary mediator driving the progression of fibrosis, glomerulosclerosis and especially mesangial cell phenotype transformation in diabetic nephropathy (DN) $[1,2]$. TGF- $\beta 1$ directly stimulates the transcription of extracellular matrix (ECM). Increased TGF- $\beta 1$ is reported to be associated with $\mathrm{DN}$ disease [3-5]. Gene polymorphisms of TGF- $\beta 1$ can affect the activity of TGF- $\beta 1$. The TGF- $\beta 1$ T869C gene polymorphism is one of the most important gene polymorphisms that affects the protein expression of TGF- $\beta 1$ [6]. Gene polymorphisms have been reported to be associated with some diseases [7-9]. However, there are conflicting reports on the association of the TGF- $\beta 1$ T869C polymorphism with T2DN susceptibility [10-13].

Diabetes mellitus (DM), characterized by elevated levels of blood glucose, is a complex and heterogeneous, chronic metabolic disease [14]. DM is the leading cause of morbidity and mortality worldwide and is a major global health problem $[15,16]$. DM is divided into four different etiological categories: type 1 DM (T1DM), type 2 DM (T2DM), other specific types, and gestational DM. The main characteristic of T2DM is insulin resistance, often followed by the failure of pancreatic $\beta$-cells. Recent data indicate that morbidity and mortality among diabetic patients are increased [14]. One severe complication of T2DM is type 2 diabetic nephropathy (T2DN), which is characterized by hypertension, albuminuria, and a progressive decline in glomerular filtration rate, developing into end-stage renal disease $[17,18]$. There is increasing evidence showing that TGF- $\beta 1$ takes part in the pathogenesis of T2DN [19-21].

In this study, we assessed the association between TGF- $\beta 1$ levels and T2DN risk, and the association of the TGF- $\beta 1$ T869C gene polymorphism with the susceptibility to T2DN in Chinese population, by a meta-analysis method.

\section{Methods}

\section{Search strategy}

The electronic databases of the Cochrane Library, the China Biological Medicine Database (CBM), and PubMed were searched on March 1, 2018, and relevant studies were retrieved. The retrieval strategy of "(transforming growth factor- $\beta 1$ OR TGF- $\beta 1$ ) AND (diabetic nephropathy OR diabetic kidney disease)" was entered and searched in these databases. Additional investigations were extracted from the references cited in articles retrieved in this search.

\section{Inclusion and exclusion criteria Inclusion criteria}

(1) Each study had at least two comparison groups (case group vs. control group); (2) The outcome in patients had to be T2DN; (3) Each study should show data on the TGF- $\beta 1$ level and/or the TGF- $\beta 1$ T869C genotype distribution.

\section{Exclusion criteria}

(1) Editorials, review articles, case reports; (2) Study results not showing the TGF- $\beta 1$ level or the TGF- $\beta 1$ T869C gene polymorphism to disease; (3) Multiple publications from the same study group; (4) Study not conducted in Chinese population.

\section{Data extraction and synthesis}

The information was extracted from each eligible report by two authors independently: the surname of the first author, the publication year, the country of the study or ethnicity, the TGF- $\beta 1$ levels, the number of patients or controls, and the number of subjects in case groups and control groups for TGF- $\beta 1$ genotypes.

\section{Statistical analysis}

Cochrane Review Manager Version 5 software (Cochrane Library, UK) was used to calculate the available data from each investigation. The fixed effects model was used to calculate the pooled statistic. However, a random effects model was used to assess the relationship when the $P$ value of the heterogeneity test was less than 0.1 . The results were expressed as weighted mean differences (MD) for continuous data, and odds ratios (OR) were used to express the results for dichotomous data. Additionally, 95\% confidence intervals $(\mathrm{CI})$ were also counted. $P<0.05$ was required for statistical significance for the pooled OR. $I^{2}$ was used to test the heterogeneity among the included investigations. The Egger regression asymmetry test [22] and the Begg adjusted-rank correlation test [23] were used to test the publication bias, and $P<0.10$ was considered significant.

\section{Results}

\section{Study characteristics}

Forty-five reports [24-68] were included for the meta-analysis of the relationship between TGF- $\beta 1$ level and T2DN risk in Chinese population (Table 1). One report [67] was published in English and other reports were published in Chinese.

Eight studies [12, 32, 69-74] reporting the association of the TGF- $\beta 1$ T869C gene polymorphism with susceptibility to T2DN were included in this study. Two report $[69,74]$ were published using the English language and the other reports were published using Chinese. The data for the pooled OR were extracted (Table 2). Those 8 investigations contained 1018 patients with T2DN and 941 controls. The average distribution frequency of the TGF- $\beta 1 \mathrm{~T}$ allele in the T2DN group in Chinese patients was $38.15 \%$ and the average frequency in the control group was $44.72 \%$. The average distribution frequency of the TGF- $\beta 1 \mathrm{~T}$ allele in the case group was lower than that in the control group in Chinese population $($ Case/Control $=0.85)$. 
Table 1 General characteristics of the included studies for TGF- $\beta 1$ levels in T2DN in this meta-analysis

\begin{tabular}{|c|c|c|c|c|c|c|c|c|}
\hline \multirow{2}{*}{$\begin{array}{l}\text { First author, } \\
\text { year }\end{array}$} & \multirow[t]{2}{*}{ Country } & \multirow{2}{*}{$\begin{array}{l}\text { According to } \\
\text { UAER or UACR }\end{array}$} & \multicolumn{3}{|l|}{ Case } & \multicolumn{3}{|l|}{ Control } \\
\hline & & & Mean & SD & $\mathrm{N}$ & Mean & SD & $\mathrm{N}$ \\
\hline \multirow[t]{3}{*}{ Ju HB 2000} & \multirow[t]{3}{*}{ China } & Normoalbuminuria & 35.02 & 6.7 & 14 & 23.95 & 8.01 & 15 \\
\hline & & Microalbuminuria & 39.31 & 5.35 & 18 & 23.95 & 8.01 & 15 \\
\hline & & Macroalbuminuria & 58.58 & 9.56 & 13 & 23.95 & 8.01 & 15 \\
\hline \multirow[t]{2}{*}{ Wang YJ 2002} & \multirow[t]{2}{*}{ China } & Normoalbuminuria & 147.03 & 22.57 & 34 & 136.97 & 37.96 & 35 \\
\hline & & Macroalbuminuria & 170.65 & 18.74 & 31 & 136.97 & 37.96 & 35 \\
\hline \multirow[t]{2}{*}{ Li WM 2004} & \multirow[t]{2}{*}{ China } & Normoalbuminuria & 58.91 & 11.03 & 46 & 47.25 & 6.22 & 48 \\
\hline & & Macroalbuminuria & 387.45 & 82.06 & 48 & 47.25 & 6.22 & 48 \\
\hline \multirow[t]{2}{*}{ Li ZJ 2004} & \multirow[t]{2}{*}{ China } & Normoalbuminuria & 146 & 22 & 36 & 131 & 36 & 40 \\
\hline & & Macroalbuminuria & 172 & 19 & 44 & 131 & 36 & 40 \\
\hline \multirow[t]{3}{*}{ Jiang ZL 2005} & \multirow[t]{3}{*}{ China } & Normoalbuminuria & 428.3 & 43.7 & 29 & 412.5 & 58.4 & 35 \\
\hline & & Microalbuminuria & 578.5 & 69.4 & 27 & 412.5 & 58.4 & 35 \\
\hline & & Macroalbuminuria & 683.4 & 84.3 & 28 & 412.5 & 58.4 & 35 \\
\hline \multirow[t]{3}{*}{ Li ZZ 2005} & \multirow[t]{3}{*}{ China } & Normoalbuminuria & 41 & 15.57 & 27 & 10.04 & 5.33 & 18 \\
\hline & & Microalbuminuria & 66.35 & 18.04 & 12 & 10.04 & 5.33 & 18 \\
\hline & & Macroalbuminuria & 53.31 & 15.64 & 18 & 10.04 & 5.33 & 18 \\
\hline \multirow[t]{3}{*}{ Zhou Y 2005} & \multirow[t]{3}{*}{ China } & Normoalbuminuria & 31.12 & 12.39 & 30 & 29.4 & 10.62 & 30 \\
\hline & & Microalbuminuria & 79.63 & 15.96 & 30 & 29.4 & 10.62 & 30 \\
\hline & & Macroalbuminuria & 136.6 & 21.45 & 30 & 29.4 & 10.62 & 30 \\
\hline \multirow[t]{3}{*}{ Jing CY 2005} & \multirow[t]{3}{*}{ China } & Normoalbuminuria & 31.16 & 14.23 & 31 & 24.58 & 12.61 & 20 \\
\hline & & Microalbuminuria & 48.2 & 18.3 & 25 & 24.58 & 12.61 & 20 \\
\hline & & Macroalbuminuria & 62.12 & 21.3 & 23 & 24.58 & 12.61 & 20 \\
\hline Wei YS 2005 & China & NR & 41.57 & 10.55 & 91 & 25.46 & 7.88 & 105 \\
\hline \multirow[t]{2}{*}{ Li HP 2006} & \multirow[t]{2}{*}{ China } & Normoalbuminuria & 147.02 & 20.57 & 108 & 131.96 & 3.84 & 120 \\
\hline & & Macroalbuminuria & 170.64 & 17.72 & 132 & 131.96 & 3.84 & 120 \\
\hline Tao SP 2006 & China & Normoalbuminuria & 147 & 23 & 28 & 132 & 36 & 25 \\
\hline & & Macroalbuminuria & 172 & 18 & 34 & 132 & 36 & 25 \\
\hline Meng T 2006 & China & Normoalbuminuria & 217.7 & 126 & 28 & 84.5 & 23.4 & 30 \\
\hline & & Microalbuminuria & 288.2 & 109.4 & 24 & 84.5 & 23.4 & 30 \\
\hline & & Macroalbuminuria & 345.5 & 118.2 & 22 & 84.5 & 23.4 & 30 \\
\hline Xie HF 2006 & China & Normoalbuminuria & 42.1 & 9.3 & 60 & 35.9 & 8.1 & 30 \\
\hline & & Macroalbuminuria & 61.8 & 11.2 & 45 & 35.9 & 8.1 & 30 \\
\hline Qian YX 2006 & China & Normoalbuminuria & 146 & 22 & 48 & 131 & 36 & 60 \\
\hline & & Macroalbuminuria & 172 & 19 & 23 & 131 & 36 & 60 \\
\hline Fu CX 2007 & China & Normoalbuminuria & 36.2 & 8.8 & 34 & 34.4 & 8.2 & 35 \\
\hline & & Microalbuminuria & 69.4 & 12.8 & 31 & 34.4 & 8.2 & 35 \\
\hline Du JW 2007 & China & Normoalbuminuria & 179.16 & 13.13 & 20 & 68.47 & 31.75 & 19 \\
\hline & & Microalbuminuria & 192.66 & 57.25 & 21 & 68.47 & 31.75 & 19 \\
\hline & & Macroalbuminuria & 582.04 & 211.25 & 20 & 68.47 & 31.75 & 19 \\
\hline Zhang WJ 2007 & China & Normoalbuminuria & 23.35 & 3.7 & 36 & 20.35 & 3.7 & 40 \\
\hline & & Microalbuminuria & 41.31 & 4.3 & 45 & 20.35 & 3.7 & 40 \\
\hline & & Macroalbuminuria & 55.28 & 6.8 & 45 & 20.35 & 3.7 & 40 \\
\hline
\end{tabular}


Table 1 General characteristics of the included studies for TGF- $\beta 1$ levels in T2DN in this meta-analysis (Continued)

\begin{tabular}{|c|c|c|c|c|c|c|c|c|}
\hline \multirow{2}{*}{$\begin{array}{l}\text { First author, } \\
\text { year }\end{array}$} & \multirow[t]{2}{*}{ Country } & \multirow{2}{*}{$\begin{array}{l}\text { According to } \\
\text { UAER or UACR }\end{array}$} & \multicolumn{3}{|l|}{ Case } & \multicolumn{3}{|c|}{ Control } \\
\hline & & & Mean & SD & $\mathrm{N}$ & Mean & SD & $\mathrm{N}$ \\
\hline \multirow[t]{3}{*}{ Lai X 2007} & \multirow[t]{3}{*}{ China } & Normoalbuminuria & 89.65 & 28.33 & 27 & 31.46 & 9.07 & 43 \\
\hline & & Microalbuminuria & 121.02 & 32.36 & 21 & 31.46 & 9.07 & 43 \\
\hline & & Macroalbuminuria & 211.69 & 69.83 & 17 & 31.46 & 9.07 & 43 \\
\hline \multirow[t]{2}{*}{ Lin YH 2007} & \multirow[t]{2}{*}{ China } & Normoalbuminuria & 97.24 & 18.6 & 19 & 58.36 & 13.72 & 23 \\
\hline & & Macroalbuminuria & 136.75 & 23.48 & 24 & 58.36 & 13.72 & 23 \\
\hline \multirow[t]{2}{*}{ Zhang SF 2007} & \multirow[t]{2}{*}{ China } & Microalbuminuria & 21.188 & 20.87 & 15 & 6.99 & 18.57 & 18 \\
\hline & & Macroalbuminuria & 13.64 & 19.44 & 16 & 6.99 & 18.57 & 18 \\
\hline \multirow[t]{3}{*}{ Zhang WK 2008} & \multirow[t]{3}{*}{ China } & Normoalbuminuria & 23.3 & 10.1 & 30 & 20.3 & 3.7 & 26 \\
\hline & & Microalbuminuria & 41.3 & 4.2 & 38 & 20.3 & 3.7 & 26 \\
\hline & & Macroalbuminuria & 88.2 & 6.8 & 32 & 20.3 & 3.7 & 26 \\
\hline \multirow[t]{2}{*}{ Wang YP 2008} & \multirow[t]{2}{*}{ China } & Normoalbuminuria & 35.4 & 7.1 & 44 & 32.5 & 6.8 & 35 \\
\hline & & Macroalbuminuria & 68.2 & 12.5 & 32 & 32.5 & 6.8 & 35 \\
\hline Zhang SB 2008 & China & NR & 121.5 & 37.2 & 36 & 55.2 & 16.8 & 30 \\
\hline \multirow[t]{3}{*}{ Li QX 2008} & \multirow[t]{3}{*}{ China } & Normoalbuminuria & 31.9 & 9.72 & 26 & 21.5 & 6.89 & 20 \\
\hline & & Microalbuminuria & 49.6 & 14.78 & 23 & 21.5 & 6.89 & 20 \\
\hline & & Macroalbuminuria & 70.3 & 26.48 & 18 & 21.5 & 6.89 & 20 \\
\hline \multirow[t]{3}{*}{ Feng SJ 2008} & \multirow[t]{3}{*}{ China } & Normoalbuminuria & 208.2 & 110 & 25 & 80.62 & 3.4 & 38 \\
\hline & & Microalbuminuria & 293.3 & 118.5 & 23 & 80.62 & 3.4 & 38 \\
\hline & & Macroalbuminuria & 263.5 & 108.2 & 18 & 80.62 & 3.4 & 38 \\
\hline \multirow[t]{2}{*}{ Zhang HM 2008} & \multirow[t]{2}{*}{ China } & Normoalbuminuria & 32.52 & 12.24 & 40 & & & \\
\hline & & Microalbuminuria & 43.61 & 20.37 & 48 & & & \\
\hline \multirow[t]{3}{*}{ Cao B 2009} & China & Normoalbuminuria & 31.2 & 5.6 & 31 & 17.4 & 3.4 & 30 \\
\hline & & Microalbuminuria & 54.9 & 7.8 & 34 & 17.4 & 3.4 & 30 \\
\hline & & Macroalbuminuria & 78.2 & 10.3 & 30 & 17.4 & 3.4 & 30 \\
\hline Li QX 2009 & China & Normoalbuminuria & 31.9 & 9.72 & 26 & 21.5 & 6.89 & 20 \\
\hline & & Microalbuminuria & 49.6 & 14.78 & 23 & 21.5 & 6.89 & 20 \\
\hline & & Macroalbuminuria & 70.3 & 26.48 & 18 & 21.5 & 6.89 & 20 \\
\hline Yang YZ 2010 & China & Normoalbuminuria & 28.59 & 3.64 & 25 & 21.07 & 3.48 & 30 \\
\hline & & Macroalbuminuria & 43.12 & 4.62 & 25 & 21.07 & 3.48 & 30 \\
\hline Feng LM 2010 & China & Normoalbuminuria & 34.2 & 7.1 & 40 & 32.8 & 6.4 & 35 \\
\hline & & Macroalbuminuria & 69.4 & 7.2 & 32 & 32.8 & 6.4 & 35 \\
\hline Wu YJ 2010 & China & NG & 172.5 & 20.4 & 30 & 125.4 & 14.6 & 28 \\
\hline Ye CF 2010 & China & Normoalbuminuria & 31.36 & 5.75 & 37 & 26.54 & 5.78 & 32 \\
\hline & & Macroalbuminuria & 58.69 & 9.87 & 37 & 26.54 & 5.78 & 32 \\
\hline Huang JW 2010 & China & Normoalbuminuria & 41.85 & 10.38 & 29 & 22.5 & 5.75 & 30 \\
\hline & & Microalbuminuria & 79.51 & 44.95 & 32 & 22.5 & 5.75 & 30 \\
\hline & & Macroalbuminuria & 118.15 & 59.38 & 28 & 22.5 & 5.75 & 30 \\
\hline Chen D 2011 & China & Normoalbuminuria & 129.16 & 27.08 & 30 & 83.32 & 30.55 & 60 \\
\hline & & Microalbuminuria & 162.97 & 98.58 & 30 & 83.32 & 30.55 & 60 \\
\hline & & Macroalbuminuria & 563.46 & 122.67 & 30 & 83.32 & 30.55 & 60 \\
\hline Li QX 2011 & China & Normoalbuminuria & 31.9 & 9.72 & 26 & 21.5 & 6.89 & 20 \\
\hline & & Microalbuminuria & 49.6 & 14.78 & 23 & 21.5 & 6.89 & 20 \\
\hline & & Macroalbuminuria & 70.3 & 26.48 & 18 & 21.5 & 6.89 & 20 \\
\hline
\end{tabular}


Table 1 General characteristics of the included studies for TGF- $\beta 1$ levels in T2DN in this meta-analysis (Continued)

\begin{tabular}{|c|c|c|c|c|c|c|c|c|}
\hline \multirow{2}{*}{$\begin{array}{l}\text { First author, } \\
\text { year }\end{array}$} & \multirow[t]{2}{*}{ Country } & \multirow{2}{*}{$\begin{array}{l}\text { According to } \\
\text { UAER or UACR }\end{array}$} & \multicolumn{3}{|l|}{ Case } & \multicolumn{3}{|l|}{ Control } \\
\hline & & & Mean & SD & $\mathrm{N}$ & Mean & SD & $\mathrm{N}$ \\
\hline \multirow[t]{2}{*}{ Zhou ZX 2011} & \multirow[t]{2}{*}{ China } & Normoalbuminuria & 33.12 & 8.16 & 50 & 32.98 & 7.83 & 50 \\
\hline & & Microalbuminuria & 49.21 & 18.11 & 56 & 32.98 & 7.83 & 50 \\
\hline \multirow[t]{2}{*}{ He Y 2012} & \multirow[t]{2}{*}{ China } & Normoalbuminuria & 147.01 & 20.98 & 48 & 131.82 & 36.01 & 60 \\
\hline & & Macroalbuminuria & 172.31 & 19.06 & 42 & 131.82 & 36.01 & 60 \\
\hline Zhang Y 2012 & China & NG & 154.8 & 7.09 & 28 & 122.84 & 6.3 & 15 \\
\hline \multirow[t]{3}{*}{ Du ZC 2013} & \multirow[t]{3}{*}{ China } & Normoalbuminuria & 18.55 & 2.67 & 20 & 8.97 & 4.087 & 18 \\
\hline & & Microalbuminuria & 19.04 & 2.87 & 20 & 8.97 & 4.087 & 18 \\
\hline & & Macroalbuminuria & 18.12 & 3.17 & 21 & 8.97 & 4.087 & 18 \\
\hline \multirow[t]{3}{*}{ Zhang WQ 2014} & \multirow[t]{3}{*}{ China } & Normoalbuminuria & 30.3 & 4.42 & 32 & 24.52 & 2.81 & 23 \\
\hline & & Microalbuminuria & 34.32 & 4.32 & 41 & 24.52 & 2.81 & 23 \\
\hline & & Macroalbuminuria & 58.31 & 5.16 & 13 & 24.52 & 2.81 & 23 \\
\hline \multirow[t]{3}{*}{ Liu S 2014} & \multirow[t]{3}{*}{ China } & Normoalbuminuria & 76.8 & 3.1 & 30 & 29.6 & 2.5 & 30 \\
\hline & & Microalbuminuria & 114.8 & 3.1 & 30 & 29.6 & 2.5 & 30 \\
\hline & & Macroalbuminuria & 135.8 & 5.9 & 30 & 29.6 & 2.5 & 30 \\
\hline Bao HL 2014 & China & Microalbuminuria & 75.4 & 9.2 & 33 & 71.2 & 11.1 & 36 \\
\hline \multirow[t]{3}{*}{ Feng R 2015} & \multirow[t]{3}{*}{ China } & Normoalbuminuria & 7.58 & 2.11 & 22 & 5.13 & 1.63 & 30 \\
\hline & & Microalbuminuria & 11.89 & 3.33 & 29 & 5.13 & 1.63 & 30 \\
\hline & & Macroalbuminuria & 24.62 & 6.62 & 35 & 5.13 & 1.63 & 30 \\
\hline \multirow[t]{3}{*}{ Lv C 2015} & \multirow[t]{3}{*}{ China } & Normoalbuminuria & 27.3 & 5.45 & 137 & 14.98 & 3.23 & 131 \\
\hline & & Microalbuminuria & 51.8 & 5.72 & 122 & 14.98 & 3.23 & 131 \\
\hline & & Macroalbuminuria & 72.97 & 6.05 & 68 & 14.98 & 3.23 & 131 \\
\hline \multirow[t]{3}{*}{ Bi FC 2016} & \multirow[t]{3}{*}{ China } & Normoalbuminuria & 5.61 & 2.08 & 21 & 1.79 & 1.64 & 20 \\
\hline & & Microalbuminuria & 8.98 & 2.26 & 20 & 1.79 & 1.64 & 20 \\
\hline & & Macroalbuminuria & 11.39 & 1.61 & 20 & 1.79 & 1.64 & 20 \\
\hline
\end{tabular}

NR: not report

Association of the TGF- $\beta 1$ level with T2DN risk

In this study, we found that the serum TGF- $\beta 1$ level in the T2DM group was higher than in the normal control group $(\mathrm{MD}=17.30,95 \% \mathrm{CI}: 12.69-21.92, P<0.00001$; Table 3 and Fig. 1). The serum TGF- $\beta 1$ level in the T2DN group

Table 2 General characteristics of the included studies on TGF$\beta 1$ T869C gene polymorphism with T2DN risk in Chinese population

\begin{tabular}{|c|c|c|c|c|c|c|c|c|c|}
\hline \multirow[b]{2}{*}{ Author, Year } & \multirow[b]{2}{*}{ Ethnicity } & \multicolumn{4}{|c|}{ Case } & \multicolumn{4}{|c|}{ Control } \\
\hline & & CC & $C T$ & $\Pi$ & total & CC & CT & $\pi$ & total \\
\hline Wong, 2003 & Asian & 27 & 26 & 5 & 58 & 24 & 24 & 17 & 65 \\
\hline Wei, 2005 & Asian & 31 & 48 & 12 & 91 & 21 & 46 & 25 & 92 \\
\hline Wei, 2008 & Asian & 94 & 128 & 58 & 280 & 72 & 142 & 66 & 280 \\
\hline Chen, 2010 & Asian & 68 & 118 & 46 & 232 & 30 & 63 & 33 & 126 \\
\hline Chai, 2009 & Asian & 19 & 19 & 14 & 52 & 27 & 26 & 5 & 58 \\
\hline Pan, 2007 & Asian & 37 & 34 & 9 & 80 & 34 & 29 & 24 & 87 \\
\hline Rao, 2011 & Asian & 14 & 25 & 6 & 45 & 13 & 32 & 8 & 53 \\
\hline Mou, 2011 & Asian & 88 & 87 & 5 & 180 & 71 & 73 & 36 & 180 \\
\hline
\end{tabular}

was higher than that in the normal control group (MD = 70.03, 95\% CI: 60.81-79.26, $P<0.00001$; Table 3 and Fig. 2). The serum TGF- $\beta 1$ level in the T2DN group was higher than in the T2DM group (MD = 56.18, 95\% CI: 46.96-65.39, $P<0.00001$; Table 3 and Fig. 3). The serum TGF- $\beta 1$ level in T2DM patients with microalbuminuria was increased compared to that in T2DM patients with normoalbuminuria $(\mathrm{MD}=22.78,95 \% \mathrm{CI}:$ 16.88-28.68, $P<0.00001$; Table 3$)$. Furthermore, the serum TGF- $\beta 1$ level in T2DM patients with macroalbuminuria was increased compared to that in T2DM patients with microalbuminuria $(\mathrm{MD}=28.47,95 \%$ CI: 21.28-35.66, $P<0.00001$; Table 3).

\section{Association between the TGF- $\beta 1$ T869C gene polymorphism and T2DN susceptibility in Chinese population}

In this meta-analysis, the TGF- $\beta 1$ T allele, TT allele and CC genotype were associated with T2DN susceptibility in Chinese population ( $\mathrm{T}$ : $\mathrm{OR}=0.74,95 \% \mathrm{CI}$ : $0.59-0.92, P=0.007$; TT: $\mathrm{OR}=0.55,95 \%$ CI: $0.31-0.96, P=0.04 ; C C: \mathrm{OR}=1.38$, 95\% CI: 1.14-1.67, $P=0.001$; Fig. 4 and Table 4). 
Table 3 Meta-analysis of the association of TGF- $\beta 1$ levels with T2DN risk in Chinese population

\begin{tabular}{|c|c|c|c|c|c|}
\hline Contrasts & Studies number & $Q$ test $P$ value & Model selected & $\mathrm{MD}(95 \% \mathrm{Cl})$ & $P$ \\
\hline DM vs. Control & 38 & $<0.00001$ & Random & $17.30(12.69,21.92)$ & $<0.00001$ \\
\hline DN vs. Control & 44 & $<0.00001$ & Random & $70.03(60.81,79.26)$ & $<0.00001$ \\
\hline DM vs. DN & 37 & $<0.00001$ & Random & $56.18(46.96,65.39)$ & $<0.00001$ \\
\hline Microalbuminuria VS. Normoalbuminuria & 26 & $<0.00001$ & Random & $22.78(16.88,28.68)$ & $<0.00001$ \\
\hline Macroalbuminuria VS. Microalbuminuria & 24 & $<0.00001$ & Random & $28.47(21.28,35.66)$ & $<0.00001$ \\
\hline
\end{tabular}

\section{Evaluation of publication bias}

There were publication biases for DM vs. control (Egger $P=$ 0.001, Begg $P=0$; Fig. 5a), DN vs. control (Egger $P=0$, Begg $P=0$; Fig. $5 \mathrm{~b}$ ), DN vs. DM (Egger $P=0$, Begg $P=0$; Fig. 5 c), microalbuminuria vs. normoalbuminuria (Egger $P=0.021$, Begg $\mathrm{P}=0$; Fig. $5 \mathrm{~d}$ ), macroalbuminuria vs. microalbuminuria in Chinese population (Egger $P=0.051$, Begg $P=0.042$; Fig. 5e). Interestingly, there was no publication bias for the association of the TGF- $\beta 1$ T869C gene polymorphism with T2DN susceptibility in Chinese population (Egger $P=0.627$, Begg $P=1.000$; Fig. 5f).

\section{Discussion}

TGF- $\beta 1$ can stimulate the transcription of extracellular matrix (ECM) proteins, and high levels of TGF- $\beta 1$ are associated with ECM accumulation, fibrosis, and

\begin{tabular}{|c|c|c|c|c|c|c|c|c|c|c|c|c|c|c|}
\hline \multirow[b]{2}{*}{ Studv or Subgroup } & \multicolumn{3}{|c|}{ DN } & \multicolumn{3}{|c|}{ Control } & \multicolumn{3}{|c|}{ Mean Difference } & \multirow{2}{*}{\multicolumn{5}{|c|}{$\begin{array}{c}\text { Mean Difference } \\
\text { IV, Random, 95\% Cl }\end{array}$}} \\
\hline & Mean & SD & Total & Mean & SD & Total & Weight & IV, Random, $95 \% \mathrm{Cl}$ & Year & & & & & \\
\hline Ju HB 2000 & 35.02 & 6.7 & 14 & 23.95 & 8.01 & 15 & $2.8 \%$ & $11.07[5.71,16.43]$ & 2000 & & & - & & \\
\hline Wang YJ 2002 & 147.03 & 22.57 & 34 & 136.97 & 37.96 & 35 & $2.3 \%$ & $10.06[-4.63,24.75]$ & 2002 & & & & & \\
\hline Li WM 2004 & 58.91 & 11.03 & 46 & 47.25 & 6.22 & 48 & $2.9 \%$ & $11.66[8.02,15.30]$ & 2004 & & & - & & \\
\hline Li ZJ 2004 & 146 & 22 & 36 & 131 & 36 & 40 & $2.4 \%$ & $15.00[1.73,28.27]$ & 2004 & & & & & \\
\hline Jing CY 2005 & 31.16 & 14.23 & 31 & 24.58 & 12.61 & 20 & $2.7 \%$ & $6.58[-0.88,14.04]$ & 2005 & & & - & & \\
\hline Zhou Y 2005 & 31.12 & 12.39 & 30 & 29.4 & 10.62 & 30 & $2.8 \%$ & $1.72[-4.12,7.56]$ & 2005 & & & - & & \\
\hline Jiang ZL 2005 & 428.3 & 43.7 & 29 & 412.5 & 58.4 & 35 & $1.6 \%$ & $15.80[-9.25,40.85]$ & 2005 & & & & & \\
\hline Li ZZZ 2005 & 41 & 15.57 & 27 & 10.04 & 5.33 & 18 & $2.8 \%$ & $30.96[24.59,37.33]$ & 2005 & & & & & \\
\hline Xie HF 2006 & 42.1 & 9.3 & 60 & 35.9 & 8.1 & 30 & $2.9 \%$ & $6.20[2.47,9.93]$ & 2006 & & & - & & \\
\hline Tao SP 2006 & 147 & 23 & 28 & 132 & 36 & 25 & $2.1 \%$ & $15.00[-1.48,31.48]$ & 2006 & & & & & \\
\hline Qian $Y \times 2006$ & 146 & 22 & 48 & 131 & 36 & 60 & $2.5 \%$ & $15.00[3.97,26.03]$ & 2006 & & & $\longrightarrow$ & & \\
\hline Li HP 2006 & 147.02 & 20.57 & 108 & 131.96 & 3.84 & 120 & $2.9 \%$ & $15.06[11.12,19.00]$ & 2006 & & & - & & \\
\hline Meng T 2006 & 217.7 & 126 & 28 & 84.5 & 23.4 & 30 & $0.7 \%$ & $133.20[85.78,180.62]$ & 2006 & & & & & $\rightarrow$ \\
\hline Du JW 2007 & 179.16 & 13.13 & 20 & 68.47 & 31.75 & 19 & $2.2 \%$ & $110.69[95.30,126.08]$ & 2007 & & & & & + \\
\hline Zhang WJ 2007 & 23.35 & 3.7 & 36 & 20.35 & 3.7 & 40 & $2.9 \%$ & $3.00[1.33,4.67]$ & 2007 & & & - & & \\
\hline Lin YH 2007 & 97.24 & 18.6 & 19 & 58.36 & 13.72 & 23 & $2.6 \%$ & $38.88[28.81,48.95]$ & 2007 & & & & & \\
\hline Fu CX 2007 & 36.2 & 8.8 & 34 & 34.4 & 8.2 & 35 & $2.9 \%$ & $1.80[-2.22,5.82]$ & 2007 & & & - & & \\
\hline Lai $\times 2007$ & 89.65 & 28.33 & 27 & 31.46 & 9.07 & 43 & $2.5 \%$ & $58.19[47.17,69.21]$ & 2007 & & & & & \\
\hline Feng SJ 2008 & 208.2 & 110 & 25 & 80.62 & 3.4 & 38 & $0.8 \%$ & $127.58[84.45,170.71]$ & 2008 & & & & & $\rightarrow$ \\
\hline Li QX 2008 & 31.9 & 9.72 & 26 & 21.5 & 6.89 & 20 & $2.9 \%$ & $10.40[5.60,15.20]$ & 2008 & & & - & & \\
\hline Wang YP 2008 & 35.4 & 7.1 & 44 & 32.5 & 6.8 & 35 & $2.9 \%$ & $2.90[-0.18,5.98]$ & 2008 & & & - & & \\
\hline Zhang WK 2008 & 23.3 & 10.1 & 30 & 20.3 & 3.7 & 26 & $2.9 \%$ & $3.00[-0.88,6.88]$ & 2008 & & & - & & \\
\hline Cao B 2009 & 31.2 & 5.6 & 31 & 17.4 & 3.4 & 30 & $2.9 \%$ & $13.80[11.48,16.12]$ & 2009 & & & - & & \\
\hline Li $Q \times 2009$ & 31.9 & 9.72 & 26 & 21.5 & 6.89 & 20 & $2.9 \%$ & $10.40[5.60,15.20]$ & 2009 & & & - & & \\
\hline Yang YZ 2010 & 28.59 & 3.64 & 25 & 21.07 & 3.48 & 30 & $2.9 \%$ & $7.52[5.63,9.41]$ & 2010 & & & - & & \\
\hline Feng LM 2010 & 34.2 & 7.1 & 40 & 32.8 & 6.4 & 35 & $2.9 \%$ & $1.40[-1.66,4.46]$ & 2010 & & & - & & \\
\hline Huang JW 2010 & 41.85 & 10.38 & 29 & 22.5 & 5.75 & 30 & $2.9 \%$ & $19.35[15.05,23.65]$ & 2010 & & & - & & \\
\hline Ye CF 2010 & 31.36 & 5.75 & 37 & 26.54 & 5.78 & 32 & $2.9 \%$ & $4.82[2.09,7.55]$ & 2010 & & & - & & \\
\hline Li QX 2011 & 31.9 & 9.72 & 26 & 21.5 & 6.89 & 20 & $2.9 \%$ & $10.40[5.60,15.20]$ & 2011 & & & - & & \\
\hline Zhou $2 \times 2011$ & 33.12 & 8.16 & 50 & 32.98 & 7.83 & 50 & $2.9 \%$ & $0.14[-2.99,3.27]$ & 2011 & & & & & \\
\hline Chen D 2011 & 129.16 & 27.08 & 30 & 83.32 & 30.55 & 60 & $2.4 \%$ & $45.84[33.44,58.24]$ & 2011 & & & & & \\
\hline He Y 2012 & 147.01 & 20.98 & 48 & 131.82 & 36.01 & 60 & $2.5 \%$ & $15.19[4.32,26.06]$ & 2012 & & & $\longrightarrow$ & & \\
\hline Du ZC 2013 & 18.55 & 2.67 & 20 & 8.97 & 4.087 & 18 & $2.9 \%$ & $9.58[7.36,11.80]$ & 2013 & & & - & & \\
\hline Liu $S 2014$ & 76.8 & 3.1 & 30 & 29.6 & 2.5 & 30 & $2.9 \%$ & $47.20[45.77,48.63]$ & 2014 & & & & - & \\
\hline Zhang WQ 2014 & 30.3 & 4.42 & 32 & 24.52 & 2.81 & 23 & $2.9 \%$ & $5.78[3.87,7.69]$ & 2014 & & & - & & \\
\hline LVC 2015 & 27.3 & 5.45 & 137 & 14.98 & 3.23 & 131 & $2.9 \%$ & $12.32[11.25,13.39]$ & 2015 & & & $\cdot$ & & \\
\hline Feng R 2015 & 7.58 & 2.11 & 22 & 5.13 & 1.63 & 30 & $2.9 \%$ & $2.45[1.39,3.51]$ & 2015 & & & 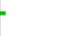 & & \\
\hline Bi FC 2016 & 5.61 & 2.08 & 21 & 1.79 & 1.64 & 20 & $2.9 \%$ & $3.82[2.68,4.96]$ & 2016 & & & - & & \\
\hline & & & 1384 & & & 1404 & $100.0 \%$ & $17.30[12.69,21.92]$ & & & & $\boldsymbol{\gamma}$ & & \\
\hline \multicolumn{10}{|c|}{$\begin{array}{l}\text { Heterogeneity: } \text { Tau }=187.74 ; \mathrm{Chi}^{2}=3609.01, \mathrm{df}=37(\mathrm{P}<0.00001) ; \mathrm{I}^{2}=99 \% \\
\text { Test for overall effect: } Z=7.35(\mathrm{P}=0.00001)\end{array}$} & -100 & Favours DM & Favou & $\begin{array}{l}50 \\
\text { urs cor }\end{array}$ & $\begin{array}{l}100 \\
\text { ntrol }\end{array}$ \\
\hline
\end{tabular}




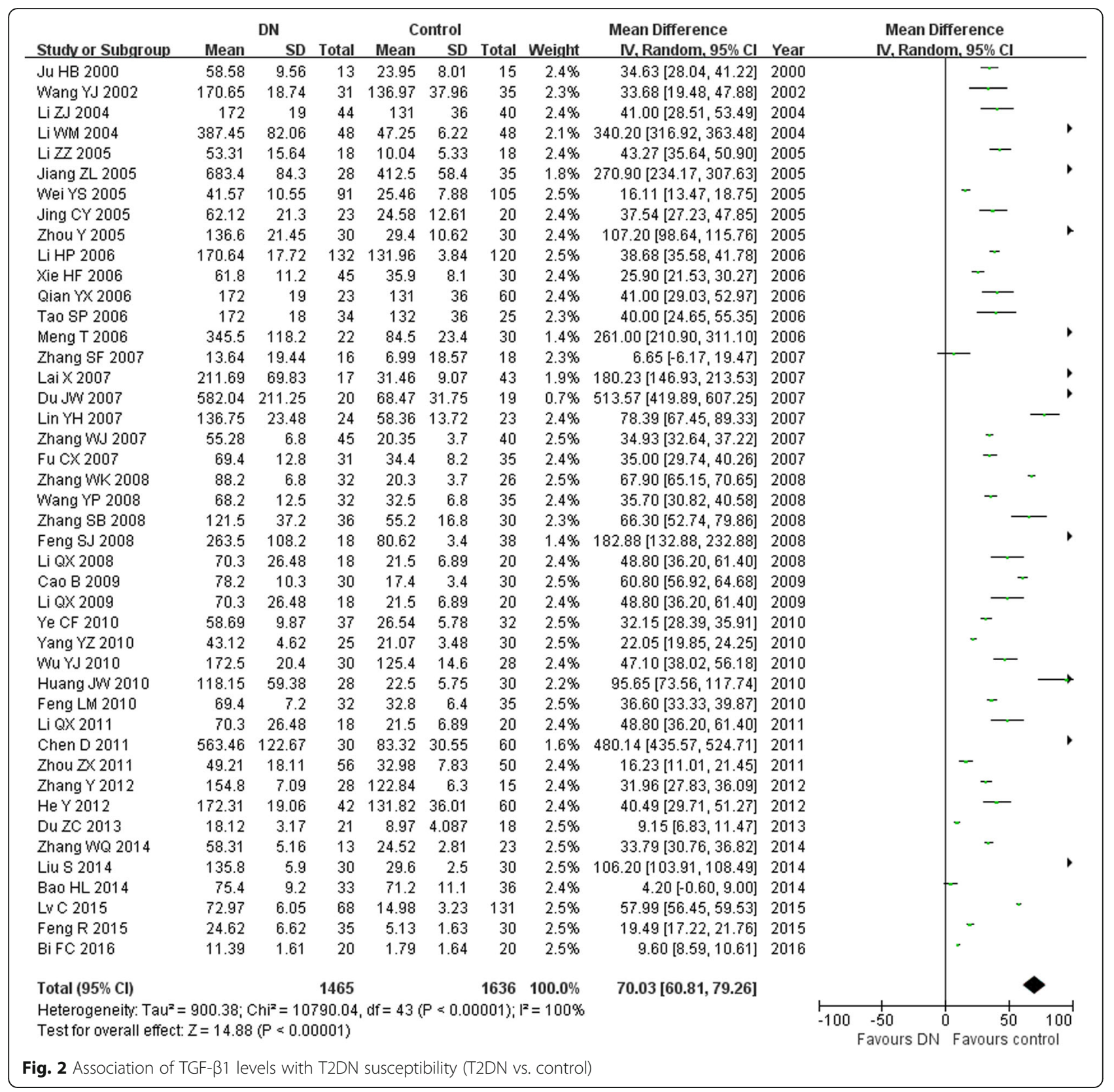

glomerulosclerosis. Glomerulosclerosis is one of most important characteristics of patients with T2DN. In this study, we performed the meta-analysis in Chinese population and found that serum levels of TGF- $\beta 1$ in the T2DM group were higher than those in the normal control group. The serum TGF- $\beta 1$ level in the T2DN group was higher than that in the normal control group or the T2DM group. Indeed, the levels of TGF- $\beta 1$ in the T2DM group and the T2DN group were higher than those in the normal control group. The level of TGF- $\beta 1$ in T2DN was higher than that in the other two groups. We also performed a subgroup analysis according to albuminuria levels. The serum TGF- $\beta 1$ level in T2DM patients with microalbuminuria was increased over that in T2DM patients with normoalbuminuria, and the serum TGF- $\beta 1$ level in T2DM patients with macroalbuminuria was increased over that in T2DM patients with microalbuminuria. This indicated that the more urine protein is, the more severe the kidney disease becomes.

Qiao et al. [75] conducted a meta-analysis based on 26 studies with 1968 cases and 2100 controls to evaluate the association between the levels of serum TGF- $\beta 1$, and urinary TGF- $\beta 1$ in patients with DM or diabetic nephropathy (DN). They reported that the levels of serum and urinary TGF- $\beta 1$ were significantly increased in T2DM and T2DN. Mou et al. [76] assessed 9 reports that included 264 patients and 227 


\begin{tabular}{|c|c|c|c|c|c|c|c|c|c|c|c|c|c|c|}
\hline \multirow[b]{2}{*}{ Study or Subgroup } & \multicolumn{3}{|c|}{ DN } & \multicolumn{3}{|c|}{ DM } & \multicolumn{2}{|r|}{ Mean Difference } & \multirow{2}{*}{\multicolumn{6}{|c|}{$\begin{array}{l}\text { Mean Difference } \\
\text { IV. Random, } 95 \% \mathrm{Cl}\end{array}$}} \\
\hline & Mean & SD & Total & Mean & SD & Total & Weight & IV, Random, $95 \% \mathrm{Cl}$ & & & & & & \\
\hline Ju HB 2000 & 58.58 & 9.56 & 13 & 35.02 & 6.7 & 14 & $3.0 \%$ & $23.56[17.29,29.83]$ & 2000 & & & - & & \\
\hline Wang YJ 2002 & 170.65 & 18.74 & 31 & 147.03 & 22.57 & 34 & $2.9 \%$ & $23.62[13.57,33.67]$ & 2002 & & & - & & \\
\hline Li ZJ 2004 & 172 & 19 & 44 & 146 & 22 & 36 & $2.9 \%$ & $26.00[16.88,35.12]$ & 2004 & & & $\longrightarrow$ & & \\
\hline Li WM 2004 & 387.45 & 82.06 & 48 & 58.91 & 11.03 & 46 & $2.5 \%$ & $328.54[305.11,351.97]$ & 2004 & & & & & - \\
\hline Zhou Y 2005 & 136.6 & 21.45 & 30 & 31.12 & 12.39 & 30 & $2.9 \%$ & $105.48[96.62,114.34]$ & 2005 & & & & & - \\
\hline Jiang ZL 2005 & 683.4 & 84.3 & 28 & 428.3 & 43.7 & 29 & $2.1 \%$ & $255.10[220.06,290.14]$ & 2005 & & & & & - \\
\hline Li ZZZ 2005 & 53.31 & 15.64 & 18 & 41 & 15.57 & 27 & $2.9 \%$ & $12.31[3.00,21.62]$ & 2005 & & & - & & \\
\hline Jing CY 2005 & 62.12 & 21.3 & 23 & 31.16 & 14.23 & 31 & $2.9 \%$ & $30.96[20.92,41.00]$ & 2005 & & & - & & \\
\hline Meng T 2006 & 345.5 & 118.2 & 22 & 217.7 & 126 & 28 & $1.1 \%$ & $127.80[59.85,195.75]$ & 2006 & & & & & $\rightarrow$ \\
\hline Xie HF 2006 & 61.8 & 11.2 & 45 & 42.1 & 9.3 & 60 & $3.0 \%$ & $19.70[15.67,23.73]$ & 2006 & & & - & & \\
\hline Qian $Y \times 2006$ & 172 & 19 & 23 & 146 & 22 & 48 & $2.9 \%$ & $26.00[16.05,35.95]$ & 2006 & & & $\longrightarrow$ & & \\
\hline Tao SP 2006 & 172 & 18 & 34 & 147 & 23 & 28 & $2.9 \%$ & $25.00[14.55,35.45]$ & 2006 & & & - & & \\
\hline Li HP 2006 & 170.64 & 17.72 & 132 & 147.02 & 20.57 & 108 & $3.0 \%$ & $23.62[18.70,28.54]$ & 2006 & & & - & & \\
\hline Zhang WJ 200 ? & 55.28 & 6.8 & 45 & 23.35 & 3.7 & 36 & $3.0 \%$ & $31.93[29.60,34.26]$ & 2007 & & & - & & \\
\hline Du JW 2007 & 582.04 & 211.25 & 20 & 179.16 & 13.13 & 20 & $0.7 \%$ & $402.88[310.12,495.64]$ & 2007 & & & & & - \\
\hline Lai $\times 2007$ & 211.69 & 69.83 & 17 & 89.65 & 28.33 & 27 & $2.1 \%$ & $122.04[87.17,156.91]$ & 2007 & & & & & $\rightarrow$ \\
\hline Lin YH 2007 & 136.75 & 23.48 & 24 & 97.24 & 18.6 & 19 & $2.9 \%$ & $39.51[26.93,52.09]$ & 2007 & & & & & \\
\hline Zhang WK 2008 & 88.2 & 6.8 & 32 & 23.3 & 10.1 & 30 & $3.0 \%$ & $64.90[60.59,69.21]$ & 2008 & & & & - & \\
\hline Feng SJ 2008 & 263.5 & 108.2 & 18 & 208.2 & 110 & 25 & $1.2 \%$ & $55.30[-10.71,121.31]$ & 2008 & & & & & \\
\hline Wang YP 2008 & 68.2 & 12.5 & 32 & 35.4 & 7.1 & 44 & $3.0 \%$ & $32.80[27.99,37.61]$ & 2008 & & & - & & \\
\hline $\mathrm{Li} Q \times 2008$ & 70.3 & 26.48 & 18 & 31.9 & 9.72 & 26 & $2.9 \%$ & $38.40[25.61,51.19]$ & 2008 & & & & & \\
\hline Cao B 2009 & 78.2 & 10.3 & 30 & 31.2 & 5.6 & 31 & $3.0 \%$ & $47.00[42.82,51.18]$ & 2009 & & & & & \\
\hline $\mathrm{Li} Q \times 2009$ & 70.3 & 26.48 & 18 & 31.9 & 9.72 & 26 & $2.9 \%$ & $38.40[25.61,51.19]$ & 2009 & & & & & \\
\hline Yang YZ 2010 & 43.12 & 4.62 & 25 & 28.59 & 3.64 & 25 & $3.0 \%$ & $14.53[12.22,16.84]$ & 2010 & & & - & & \\
\hline Ye CF 2010 & 58.69 & 9.87 & 37 & 31.36 & 5.75 & 37 & $3.0 \%$ & $27.33[23.65,31.01]$ & 2010 & & & - & & \\
\hline Huang JW 2010 & 118.15 & 59.38 & 28 & 41.85 & 10.38 & 29 & $2.6 \%$ & $76.30[53.98,98.62]$ & 2010 & & & & & \\
\hline Feng LM 2010 & 69.4 & 7.2 & 32 & 34.2 & 7.1 & 40 & $3.0 \%$ & $35.20[31.87,38.53]$ & 2010 & & & - & & \\
\hline Zhou ZX 2011 & 49.21 & 18.11 & 56 & 33.12 & 8.16 & 50 & $3.0 \%$ & $16.09[10.84,21.34]$ & 2011 & & & - & & \\
\hline Chen D 2011 & 563.46 & 122.67 & 30 & 129.16 & 27.08 & 30 & $1.8 \%$ & $434.30[389.35,479.25]$ & 2011 & & & & & - \\
\hline Li $Q \times 2011$ & 70.3 & 26.48 & 18 & 31.9 & 9.72 & 26 & $2.9 \%$ & $38.40[25.61,51.19]$ & 2011 & & & & & \\
\hline $\mathrm{HeY} 2012$ & 172.31 & 19.06 & 42 & 147.01 & 20.98 & 48 & $2.9 \%$ & $25.30[17.03,33.57]$ & 2012 & & & - & & \\
\hline Du ZC 2013 & 18.12 & 3.17 & 21 & 18.55 & 2.67 & 20 & $3.0 \%$ & $-0.43[-2.22,1.36]$ & 2013 & & & & & \\
\hline Liu S 2014 & 135.8 & 5.9 & 30 & 76.8 & 3.1 & 30 & $3.0 \%$ & $59.00[56.62,61.38]$ & 2014 & & & & - & \\
\hline Zhang WQ 2014 & 58.31 & 5.16 & 13 & 30.3 & 4.42 & 32 & $3.0 \%$ & $28.01[24.81,31.21]$ & 2014 & & & - & & \\
\hline Feng R 2015 & 24.62 & 6.62 & 35 & 7.58 & 2.11 & 22 & $3.0 \%$ & $17.04[14.68,19.40]$ & 2015 & & & - & & \\
\hline LVC 2015 & 7.58 & 2.11 & 22 & 27.3 & 5.45 & 137 & $3.0 \%$ & $-19.72[-20.99,-18.45]$ & 2015 & & - & & & \\
\hline Bi FC 2016 & 11.39 & 1.61 & 20 & 5.61 & 2.08 & 21 & $3.0 \%$ & $5.78[4.64,6.92]$ & 2016 & & & - & & \\
\hline \multicolumn{3}{|l|}{ Total $(95 \% \mathrm{Cl})$} & \multicolumn{2}{|l|}{1154} & & \multicolumn{2}{|c|}{$1350 \quad 100.0 \%$} & $56.18[46.96,65.39]$ & & \multirow[b]{2}{*}{-100} & & & & \\
\hline \multicolumn{8}{|c|}{$\begin{array}{l}\text { Heterogeneity: } \mathrm{Tau}^{2}=732.30 ; \mathrm{Chi}^{2}=7737.29, \mathrm{df}=36(\mathrm{P}<0.00001) ; \mathrm{l}^{2}=100 \% \\
\text { Test for overall effect: } Z=11.95(\mathrm{P}<0.00001)\end{array}$} & & & & $\begin{array}{c}1 \\
-50 \\
\text { Favours DN }\end{array}$ & $\begin{array}{lr}0 & 5 \\
\text { Favours }\end{array}$ & & 100 \\
\hline
\end{tabular}

healthy controls in a meta-analysis to study the relationship between serum TGF- $\beta 1$ levels and the risk of diabetic nephropathy. Their study indicated that increased serum TGF- $\beta 1$ levels in DM patients were associated with a high risk of renal involvement. The results from Qiao et al. and Mou et al. indicated that serum and urinary TGF- $\beta 1$ were significantly increased in DM and DN. Our meta-analysis included 45 reports to study the relationship between TGF- $\beta 1$ level and T2DN risk in Chinese population. Our study concludes that high levels of TGF- $\beta 1$ are associated with the susceptibility to T2DM, T2DN, and the progression of proteinuria in T2DN patients in Chinese population.

The association of the TGF- $\beta 1$ T869C gene polymorphism with the risk of $\mathrm{T} 2 \mathrm{DN}$ in Chinese population was also assessed. In this meta-analysis, we found that TGF- $\beta 1 \mathrm{~T}$ allele, and TT genotype were protective factors against the onset of T2DN in Chinese population and CC genotype was a risk factor for the susceptibility of T2DN in Chinese populations. There was no publication bias for this meta-analysis. The results might be robust to some extent. However, there were only eight studies included into for this meta-analysis in Chinese population and more number of studies should be conducted to confirm the validity of these conclusions in the future.

In a previous study, Jia et al. [77] conducted a meta-analysis to evaluate the impact of the TGF- $\beta 1$ T869C gene polymorphism on $\mathrm{DN}$, and reported that the TGF- $\beta 1$ T $869 \mathrm{C}$ gene polymorphism was associated with an elevated risk of DN disease. However, this notable association was observed only in T2DM patients. Zhou et al. [78] conducted a meta-analysis and indicated that the TGF- $\beta 1$ CC genotype was associated with T2DN risk, and that the TGF- $\beta 1 \mathrm{~T}$ allele and the CC genotype were associated with the susceptibility to T2DN. In this meta-analysis, we firstly conducted the meta-analysis in Chinese population and observed that 


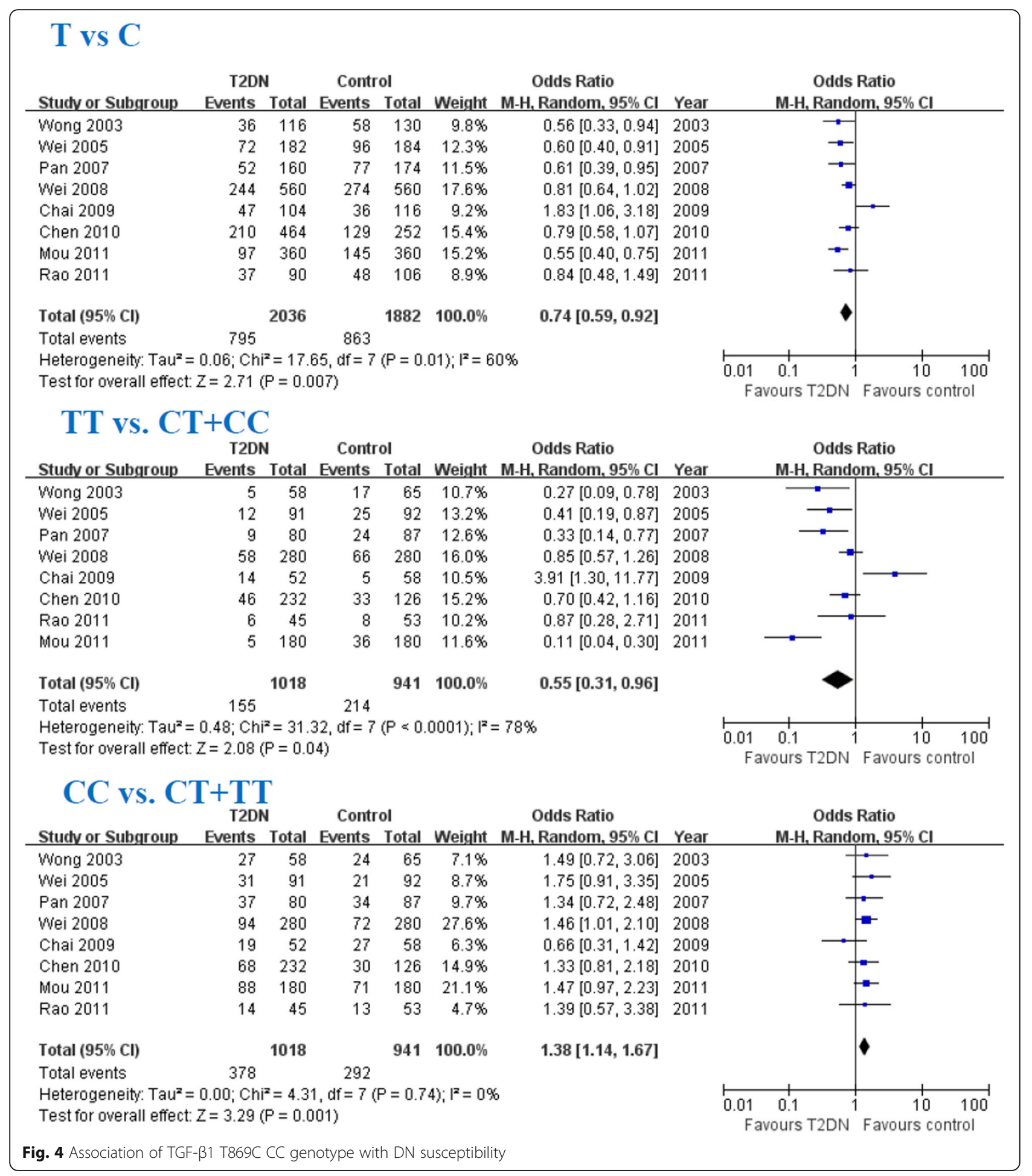

Table 4 Meta-analysis of the association of TGF- $\beta 1$ T869C gene polymorphism with T2DN risk in Chinese population

\begin{tabular}{lllll}
\hline Genetic contrasts & Studies number & Q test $P$ value & Model selected & OR $(95 \% \mathrm{Cl})$ \\
\hline CC vs. CT+TT & 8 & 0.74 & Fixed & $1.38(1.14,1.67)$ \\
TT vs. CT + CC & 8 & $<0.00001$ & Random & $0.55(0.31,0.96)$ \\
T vs. C & 8 & 0.01 & Random & 0.001 \\
\hline
\end{tabular}



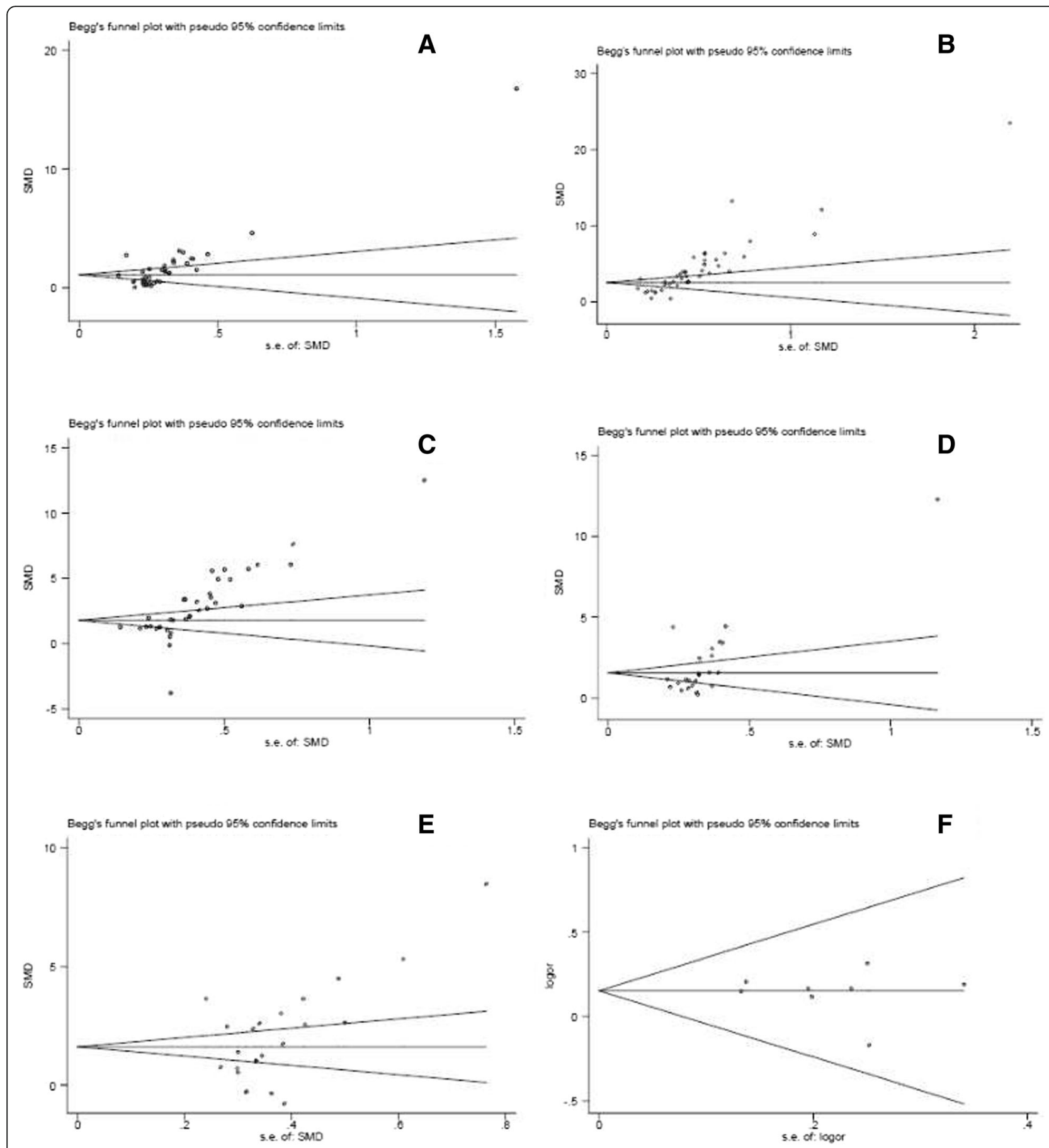

Fig. 5 Publication bias. a DM vs. control. b DN vs. control. c DN vs. DM. d microalbuminuria vs. normoalbuminuria. e macroalbuminuria vs. microalbuminuria. $\mathbf{f}$ the association of the TGF- $\beta 1$ T869C gene polymorphism with T2DN susceptibility in Chinese population

the TGF- $\beta 1$ T allele, TT genotype and CC genotype are associated with the susceptibility to T2DN in Chinese population. However, more studies are also needed to confirm this in the future.

The conclusions of our meta-analysis are limited because of the nature of the studies we analyzed. The studies themselves had several limitations, such as publication bias (most of the included studies from Chinese populations), heterogeneity of enrolled cases, small sample sizes, varying levels of plasma protein in different studies and different samples, and different timelines. In this meta-analysis, we conducted a subgroup analysis to delete any study with small sample size (less than 100), and we found that in the meta-analysis of only the larger sample studies, the $\mathrm{CC}$ genotype was 
associated with T2DN susceptibility (data not shown). However, the TGF- $\beta 1$ T869C gene polymorphism was not associated with T2DN susceptibility in the meta-analysis that included small sample size studies (data not shown). In this study, we also found that there were publication biases among the recruited investigations for the relationship between serum TGF- $\beta 1$ levels and the risk of T2DN, and for the relationship between the TGF- $\beta 1$ T869C gene polymorphism and the risk of T2DN.

\section{Conclusions}

In conclusion, this study indicated that the serum TGF- $\beta 1$ level in T2DM patients with microalbuminuria was significantly increased over that in T2DM patients with normoalbuminuria in Chinese population. The serum TGF- $\beta 1$ level in T2DM patients with macroalbuminuria was significantly increased over that in T2DM patients with microalbuminuria in Chinese population. Furthermore, the TGF- $\beta 1 \mathrm{~T}$ allele, TT genotype and $\mathrm{CC}$ genotype are associated with the susceptibility to T2DN in Chinese population. However, more association studies are required to confirm the relationships.

\section{Abbreviations}

DM: Diabetes mellitus; DN: diabetic nephropathy; ECM: extracellular matrix; T2DN: type 2 diabetic nephropathy; TGF- $\beta 1$ : transforming growth factor- $\beta 1$

\section{Acknowledgements}

Not applicable.

\section{Funding}

This study was supported by the Guangzhou Medical Key Discipline Construction Project.

\section{Availability of data and materials}

The datasets used and/or analyzed during the current study are available from the corresponding author on reasonable request.

\section{Authors' contributions}

TBZ was in charge of conceiving and designing the study. HYL and HZZ were responsible for the collection of data and performing the statistical analysis and manuscript preparation. $\mathrm{HZZ}$ and ZQZ were responsible for checking the data. All authors were responsible for drafting the manuscript, reading it, and approving the final version.

\section{Ethics approval and consent to participate}

Not applicable.

\section{Consent for publication}

Not applicable.

\section{Competing interests}

The authors declare that they have no competing interests.

\section{Publisher's Note}

Springer Nature remains neutral with regard to jurisdictional claims in published maps and institutional affiliations.

\section{Author details}

'Department of Nephrology, the Second Affiliated Hospital of Shantou University Medical College, No 69 Dongsha Road, Shantou 515041, China. ${ }^{2}$ Department of Nephrology, Huadu District People's Hospital of Guangzhou, Southern Medical University, Guangzhou 510800, China.
Received: 20 June 2018 Accepted: 14 November 2018

Published online: 20 November 2018

\section{References}

1. Liling Y, Qian Z, Qiong W, Yi W, Jiawei Y, Jiao M, Jun Z, Wei Z, Bing F. Effect of TET2 on the pathogenesis of diabetic nephropathy through activation of transforming growth factor beta1 expression via DNA demethylation. Life Sci. 2018;207:127-37.

2. Lawson JS, Syme HM, Wheeler-Jones CPD, Elliott J. Characterisation of feline renal cortical fibroblast cultures and their transcriptional response to transforming growth factor beta1. BMC Vet Res. 2018;14(1):76.

3. Shi GJ, Shi GR, Zhou JY, Zhang WJ, Gao CY, Jiang YP, Zi ZG, Zhao HH, Yang $Y, Y u$ JQ. Involvement of growth factors in diabetes mellitus and its complications: a general review. Biomed Pharmacother. 2018;101:510-27.

4. Sun J, Wang Y, Cui W, Lou Y, Sun G, Zhang D, Miao L. Role of epigenetic histone modifications in diabetic kidney disease involving renal fibrosis. J Diabetes Res. 2017;2017:7242384

5. Zhou SX, Huo DM, He XY, Yu P, Xiao YH, Ou CL, Jiang RM, Li D, Li H. High glucose/lysophosphatidylcholine levels stimulate extracellular matrix deposition in diabetic nephropathy via plateletactivating factor receptor. Mol Med Rep. 2018;17(2):2366-72.

6. Khaali W, Moumad K, Ben Driss EK, Benider A, Ben Ayoub W, Hamdi-Cherif M, Boualga K, Hassen E, Corbex M, Khyatti M. No association between TGFbeta1 polymorphisms and risk of nasopharyngeal carcinoma in a large north African case-control study. BMC Med Genet. 2016;17(1):72.

7. Peng HB, Zahary MN, Tajudin LS, Lin CL, Teck CM, Sidek MR, Zulkifli A, Zilfalil BA. A novel single nucleotide polymorphism, IVS2 -97A>T, in the prostaglandin F2alpha receptor gene was identified among the Malaysian patients with glaucoma. Kobe J Med Sci. 2007:53(1-2):49-52.

8. Hollox EJ, Hoh BP. Human gene copy number variation and infectious disease. Hum Genet. 2014;133(10):1217-33.

9. Read SA, O'Connor KS, Suppiah V, Ahlenstiel CLE, Obeid S, Cook KM, Cunningham A, Douglas MW, Hogg PJ, Booth D, et al. Zinc is a potent and specific inhibitor of IFN-lambda3 signalling. Nat Commun. 2017:8:15245.

10. Buraczynska M, Baranowicz-Gaszczyk I, Borowicz E, Ksiazek A. TGF-beta1 and TSC-22 gene polymorphisms and susceptibility to microvascular complications in type 2 diabetes. Nephron Physiol. 2007;106(4):69-75.

11. Raina P, Sikka R, Kaur R, Sokhi J, Matharoo K, Singh V, Bhanwer AJ. Association of Transforming Growth Factor Beta-1 (TGF-beta1) genetic variation with type 2 diabetes and end stage renal disease in two large population samples from North India. OMICS. 2015;19(5):306-17.

12. Pan $S$, Han $X$, Yang L, Lin J, Cong X. Association of a $29 T \rightarrow C$ polymorphism of the TGF- $\beta 1$ gene with genetic susceptibility to type 2 diabetic nephropathy. Chin J Diabetes. 2007;15(7):416-7.

13. Valladares-Salgado A, Angeles-Martinez J, Rosas M, Garcia-Mena J, Utrera-Barillas D, Gomez-Diaz R, Escobedo-de la Pena J, Parra EJ, Cruz M. Association of polymorphisms within the transforming growth factor-betal gene with diabetic nephropathy and serum cholesterol and triglyceride concentrations. Nephrology (Carlton). 2010;15(6):644-8.

14. Severino P, D'Amato A, Netti L, Pucci M, De Marchis M, Palmirotta R, Volterrani M, Mancone M, Fedele F. Diabetes mellitus and ischemic heart disease: the role of ion channels. Int J Mol Sci 2018; 19(3): E802.

15. Pourmand A, Mazer-Amirshahi M, Caggiula A, Nawab A, Payette C, Johnson S. Targeted glycemic control for adult patients with type 2 diabetes mellitus in the acute care setting. Can J Diabetes. 2018;S1499-2671(17):30807-9.

16. Aynalem S, Zeleke A. Prevalence of diabetes mellitus and its risk factors among individuals aged 15 years and above in Mizan-Aman town, Southwest Ethiopia, 2016: a cross sectional study. Int J Endocrinol. 2018;2018:9317987.

17. Mansouri M, Zniber A, Boualla L, El Badaoui G, Benkacem M, Rifai K, Chraibi A, Benamar L, Sefiani A, Bayahia R. Associations between clinical characteristics and angiotensin-converting enzyme gene insertion/deletion polymorphism in Moroccan population with Type-2 diabetic nephropathy. Saudi J Kidney Dis Transpl. 2017;28(2):261-7.

18. Sadighi A, Safa J, Vatankhah AM, Ghorashi S, Aharilahagh A, Davari-Farid S, Nezami-Nargabad O, Naghavi-Behzad M, Piri R, Pishahang P, et al. Shortterm effects of lovastatin therapy on proteinuria of type 2 diabetic nephropathy: a clinical trial study. Niger Med J. 2016;57(5):253-9.

19. Deshpande S, Abdollahi M, Wang M, Lanting L, Kato M, Natarajan R. Reduced autophagy by a microRNA-mediated signaling Cascade in diabetes-induced renal glomerular hypertrophy. Sci Rep. 2018;8(1):6954. 
20. Pandey A, Goru SK, Kadakol A, Malek V, Sharma N, Gaikwad AB. H2AK119 monoubiquitination regulates angiotensin $\|$ receptor mediated macrophage infiltration and renal fibrosis in type 2 diabetic rats. Biochimie. 2016;131:68-76.

21. Zhang S, Xu H, Yu X, Wu Y, Sui D. Metformin ameliorates diabetic nephropathy in a rat model of low-dose streptozotocin-induced diabetes. Exp Ther Med. 2017;14(1):383-90.

22. Egger M, Davey Smith G, Schneider M, Minder C. Bias in meta-analysis detected by a simple, graphical test. BMJ. 1997;315(7109):629-34.

23. Begg CB, Mazumdar M. Operating characteristics of a rank correlation test for publication bias. Biometrics. 1994;50(4):1088-101.

24. Ju H, Gan P, Li J, Gao L, Shen F, Shu Z. The changes of serum TGF $\beta 1$ in diabetic nephropathy patients and clinical significances. Chin J Diabetes. 2000;8(6):336-8,69.

25. Wang Y, Han P, Lu L. Determination and clinical significance analysis of IL-6, TNF- $\alpha$ and TGF- $\beta 1$ in the patients with diabetic nephropathy. Chin J Immun. 2002;18(3):214-5.

26. Li W, Xu K, XU Y, He Y. Contents of transforming growth factor- $\beta 1$ in vivo for the diabetic nephropathy: determination and clinical significance. J Xianning Coll. 2004;18(1):30-2.

27. Li Z, Lei J, Zhong F, Chen W. Clinical significance of level changes of TGF- $\beta 1$ and IV collagens and therapeutic effects of simvastatin in patients with diabetic nephropathy. J Fourth Mil Med Univ. 2004;25(10):942-4.

28. Jiang Z, Jiang G. Application of serum TGF- $\beta 1$ determination for early diagnosis of diabetic nephropathy. J Radioimmunol. 2005;18(3):195-6.

29. Li Z, Li L, Xu R, Dong Y. Clinical study of serum matrix metalloproteinase-9 and transforming growth factor-b1 related with type 2 diabetic patients with nephropathy. J Taishan Med Coll. 2005;26(6):530-4.

30. Zhou Y, Su X, Ke S. Relationship between serum levels of transforming growth factor-b1, tumor necrosis factor-band diabetic nephropathy. Acta Med Univ Sci Technol Huazhong 2005; 34(4):441-4.

31. Jing $C, Y e S, C h e n Y, B L R$. The change of serum and urinary transforming growth factor-beta-1 and their clinical significances in type 2 diabetics. Chin J Lab Diagn. 2005;9(2):286-9.

32. Wei $Y$, Lan $Y$, You Y, Tang R, Huang Y, Zhang L, Luo B. The serum level and the genotype of TGF-b1 in patients with type 2 diabetic nephropathy. Chin J Lab Med. 2005;128(2):173-8.

33. Li H, Li D, Li X. The changes of serum IL-6 and TGF $\beta 1$ levels in diabetic nephropathy patients and the therapeutic effects of rosuvastatin on patients with diabetic nephropathy. Chin J Misdiagn. 2006;6(1):3-5.

34. Tao $S$, Chen $X$, Sun $Y$, Hang $Y$, Zhou B. Effect and its significance of astragalus injection on blood transforming growth factor- $\beta 1$ and type IV collagen in patients with diabetic nephropathy Chinese journal of integrated traditional and Western medicine on. Nephrology. 2006;7(3):156-7.

35. Meng T. Relationship between urinary microproteins and serum MDA, TGF- $\beta 1$ in patients with early diabetic nephropathy. J Pract Med. 2006;22(4):399-400.

36. Xie H, Peng L. Clinical significance of determination of serum collagen typelV (IV-C) and transforming growth factor Beta1 (TGF- $\beta 1$ ) levels in patients with diabetic nephropathy. J Radioimmunol. 2006;19(5):370-1.

37. Qian Y, Peng W, Wang H. Clinical significance of level changes of TGF- $\beta 1$ and therapeutic effects of provastatin in patients with diabetic nephropathy. J Pract Diagn Ther. 2006;20(2):117-9.

38. Fu C. Clinical significance of determination of serum cystatin $C$, transforming growth factor beta1(TGF-b1) and urine microalbumin levels in patients with DM2 nephropathy. J Radionimmunol. 2007;20(5):471-4.

39. Du J, Wu T, Nan G, Su S. The level about the TGF-b1 and proto-oncogene proteins C-sis in peripheral blood of T2DM patients with nephropathy and the clinical applications. Clin Focus. 2007;22(11):793-4.

40. Zhang W, Lin K. Association between changes in transforming growth factor-beta1 level and type 2 diabetic nephropathy. J Clin Rehabil Tissue Eng Res. 2007;11(49):9838-42.

41. Lai X, Yu A. Value of detection of TGF- $\beta 1$ in early diagnosis of nephropathy due to type 2 diabetes. Chin Trop Med. 2007;7(4):543-4.

42. Lin $Y$, Huang $X$. Influence of atorvastatin combined with injection of sodium tanshinone II a sulfonat on levels of TGF- $\beta 1$ and CTGF of patients with diabetic nephropathy. J Clin Res. 2007;24(12):2049-51.

43. Zhang S, Wang L, Li Y. Change of TGF- $\beta 1$ in patients with diabetic nephropathy and it's intervention with valsartan. Shandong Med J. 2007;47(1):21-2.

44. Zhang W. Clinical significance of determination of transforming growth factor beta1, tumor necrosis factor alpha and urine microalbumin levels in patients with DM2 nephropathy. J Clin Res. 2008;25(6):972-4.
45. Wang Y. Clinical significance of determination of changes of plasma ET and serum TGF- $\beta 1$, VEGF levels in patients with 2-type diabetes. J Radioimmunol. 2008;21(6):529-31.

46. Zhang S. Clinical significance of determination of serum TGF- $\beta 1$ and adiponectin levels in type 2 diabetics with nephropathy. J Radioimmunol. 2008;21(2):115-6.

47. Li Q, Shi F. The change of serum TGF- $\beta 1$ and its clinical significance in type 2 diabetics nephropathy. Inner Mongolia Med J. 2008;40(9):1164-5.

48. Feng S. Diagnosis of early DN diagnosis with serum MDA and TGF- $\beta 1$ levels. J Math Med. 2008;21(3):298-9.

49. Zhang $H$, Liu L, Yang $Y$, Dong $H$. Determination and intervention with simvastatin of serum transforming growth factor $\beta 1$, IV collagens levels in early diabetic nephropathy. Hei long Jiang Med J. 2008;32(4):256-7.

50. Cao B, Li S, Xiao Z. Clinical significance of combined detection of serum CRP, SIL-2R, IL-6 and TGF- $\beta 1$ levels for diagnosis of diabetic kidney disease. Chin J Heal Care Med. 2009;11(5):356-8.

51. Li QS, Meng FY. Changes and significance of blood TGF- $\beta 1$ and CTGF in type 2 diabetic nephropathy. J Pract Diabetol. 2009;5(6):32-3.

52. Yang Y, Liu L, Wang D, Du F, Feng K. Study on the relationship between amplitude of glycemic excursion and development of diabetic nephropathy as well as serum TGF- $\beta 1$ levels in patients with type2 diabetes mellitus. J Radioimmunol. 2010;23(3):326-7.

53. Feng L, Li S. Clinical significance of measurement of changes of plasma ET-1 and serum TGF- $\beta 1$, urinary microalbuminuria (MAU) levels in patients with type II diabetes. J Radioimmunol. 2010;23(3):274-5.

54. Wu Y. Effect of captopril on serum TGF- $\beta 1$ and TNF-a levels in patients with early diabetic nephropathy. Mod Med Heal. 2010;26(24):3731-3.

55. Ye C, Wang X. Changes of TGF- $\beta 1$ and RAAS in the type 2 diabetic nephropathy patients complicating with hypertension. Chin J Mod Med. 2010;20(1):77-9.

56. Huang J, Jin X. The correlation of serum TGF- $\beta 1$ and urinary type IV collagen in diabetic nephropathy. Chin J Mod Med. 2010;20(9):1296-8.

57. Chen D, Duan W, Liu J, Liu W. Correlation of serum TGF- $\beta 1$ andPDGF-BB with albumin excretion rate in patients with type 2 diabetic nephropathy. Acta Academiae Medicinae Qingdao Universitatis. 2011;47(5):450-2,5.

58. Li Q, Tian G. The change of serum ADM TGF- $\beta 1$ and its clinical significance in type II diabetics nephropathy. Inner Mongolia Med J. 2011;43(11):1294-6.

59. Zhou Z, Song Y, Lin Q. Changes of urine protein and serum TGF- $\beta 1$ in the early stage of diabetic nephropathy. Jilin Med J. 2011;32(34):7235-6.

60. He Y. Changes of serum TGF $\beta 1$ and IV collagen levels in patients with diabetic nephropathy and their clinical significance. Med Innov China. 2012;9(6):24-5.

61. Zhang Y, Wu Y. Effect of triptergium wilfordii polyglucoside on level of serum TGF- $\beta 1$, BMP-7 and gremlin in patients with diabetic nephropathy. Anhui Med J. 2012;33(12):1616-9.

62. Du Z, Zhao Y. Association of serum transforming growth factor-(TGF-b1), type IV collagen and laminin with diabetic nephropathy in type 2 diabetic patients. Heilongjiang Med J. 2013;26(3):402-7.

63. Zhang W, Wang D, Zhang J, Li P. Early diagnostic value of TGF- $\beta 1$ for diabetic nephropathy. Chin Prac Med. 2014;9(30):10-1.

64. Liu S, Cao W, Wang Z. The significance of pigment epithelium-derived factor and TGF- $\beta 1$ in early diagnosis of diabetic nephropathy. Heb Med J. 2014;36(12):1783-5.

65. Bao HL, Ye SH, Lou SX, Lu XW, Zhou XF. Infect of pingshen decoction on serum HGF, Cys $C$ and TGF-beta1 diabetic nephropathy in early stage. Zhongguo Zhong Yao Za Zhi. 2014;39(6):1128-31.

66. Feng R, Li L, Wang N, Tian J, Sun N, Liu T, Liu J. Effects of valsartan on serum transforming growth factor- $\beta 1$, D-dimer, cystatin $C$ levels in early diabetic nephropathy. Chin J Drug Eval. 2015;32(5):280-3.

67. LV C, Zhou YH, Wu C, Shao Y, Lu CL, Wang QY. The changes in miR-130b levels in human serum and the correlation with the severity of diabetic nephropathy. Diabetes Metab Res Rev. 2015;31(7):717-24.

68. Bi F, Bao L, Luo H, Gao Y, Li L, Zheng Y. The relationship of serum TGF- $\beta 1$ expression and albuminuria in type 2 diabetic nephropathy. Ningxia Med J. 2016; 38(3):199-201.

69. Wong TY, Poon P, Chow KM, Szeto CC, Cheung MK, Li PK. Association of transforming growth factor-beta (TGF-beta) T869C (Leu 10Pro) gene polymorphisms with type 2 diabetic nephropathy in Chinese. Kidney Int. 2003;63(5):1831-5.

70. Wei M, Lu Y, Li S, She Y. Study of relationship between TGF- $\beta$ Leu 10 Prolymorphism and diabetic nephropathy in type 2 diabetes. Chin. J Diabetes. 2008;16(9):538-40.

71. Chen J, Li X, Wei J, Deng Y, Li K, Tang L. Association of the TGF- $\beta 1$ gene promoter $-869 \mathrm{C} / \mathrm{T}$ polymorphisms with diabetic nephropathy in type 2 diabetes. J Pract Med. 2010;26(22):4087-9. 
72. Chai W, Liu H, Yan S. Association of transforming growth factor- $\beta 1$ gene polymorphism with diabetic nephropathy susceptibility. Chin Modern Med. 2009;16(22):33-4.

73. Rao W, Jian $\mathrm{X}$, Zhou W. The relationship between transforming growth factor- $\beta 1$ gene polymorphism and diabetic nephropathy risk in Guangdong Han population. Int Med Health Guidance News. 2011;17(6):654-6.

74. Mou X, Liu WH, Zhou DY, Liu YH, Hu YB, Ma GL, Shou CM, Chen JW, Zhao JX. Association of Chinese medicine constitution susceptibility to diabetic nephropathy and transforming growth factor-beta1 (T869C) gene polymorphism. Chin J Integr Med. 2011;17(9):680-4.

75. Qiao YC, Chen YL, Pan YH, Ling W, Tian F, Zhang XX, Zhao HL. Changes of transforming growth factor beta 1 in patients with type 2 diabetes and diabetic nephropathy: a PRISMA-compliant systematic review and metaanalysis. Medicine (Baltimore). 2017;96(15):e6583.

76. Mou X, Zhou DY, Ma JR, Liu YH, Chen HP, Hu YB, Shou CM, Chen JW, Liu WH, Ma GL. Serum TGF-beta1 as a biomarker for type 2 diabetic nephropathy: a meta-analysis of randomized controlled trials. PLoS One. 2016;11(2):e0149513.

77. Jia H, Yu L, Gao B, Ji Q. Association between the T869C polymorphism of transforming growth factor-beta 1 and diabetic nephropathy: a metaanalysis. Endocrine. 2011;40(3):372-8.

78. Zhou TB, Jiang ZP, Qin YH, Drummen GP. Association of transforming growth factor-beta1 T869C gene polymorphism with diabetic nephropathy risk. Nephrology (Carlton). 2014;19(2):107-15.

Ready to submit your research? Choose BMC and benefit from:

- fast, convenient online submission

- thorough peer review by experienced researchers in your field

- rapid publication on acceptance

- support for research data, including large and complex data types

- gold Open Access which fosters wider collaboration and increased citations

- maximum visibility for your research: over $100 \mathrm{M}$ website views per year

At $\mathrm{BMC}$, research is always in progress.

Learn more biomedcentral.com/submissions 